\title{
How e-mail raises the spectre of a digital Dark Age
}

\author{
Records can be wiped out by a click of the mouse or rendered unreadable by upgrades.
}

Sir - Throughout the ages, historical documents have been carved in stone, etched in clay, written on parchment and printed on paper. Examination of traditional documents requires merely the ability to read and understand the language or code in which they were written. Saving them requires but a thoughtfully selected repository, with a security device if needed. But the term "read" has a completely different connotation when referring to a floppy disk or a computer hard drive. So, whereas the Rosetta Stone only required interpretation of the written key, today's equivalents require all the correct operating systems and software applications.

Faced with these complexities, it is all too tempting to dump records in the recycling bin. It is nothing short of terrifying to contemplate the probable magnitude of lost records as yesterday's electronic storage devices become incompatible with today's software applications.

These problems are most alarming when considered in the context of electronic mail. Worldwide, the number of corporate mailboxes is projected to grow from 131 million in 2001 to 225 million in 2005; daily e-mail traffic is anticipated to grow from 9.7 billion pieces in 2000 to more than 35 billion in 2005 .

Little of this staggering volume is translated to hard copy. Even formal documents are frequently discarded by simply erasing them to avoid the tedium and expense of printing and archiving, on the erroneous assumption that previous iterations are of no value. The threat to the existence of hard-copy records of e-mail will become more acute as younger generations become less culturally and educationally attuned to their importance for the preservation of historical records.

A perfect electronic world should not require hard copies of e-mail (or any other electronic document). E-technology should have addressed this problem as it evolved. But as individuals, institutions and corporations have continually 'upgraded', it has become more problematic to preserve e-documents because formats, servers and institutional IT policies change. The US National Archives has recently acknowledged the lack of robust e-mail archiving solutions, but has determined that long-term storage of electronic communications must be independent of these variables (see www.archives.gov/records_management).

Institutional policies also negatively affect long-term retention of e-mail. With limits of hard-drive space on mail servers, institutional users are routinely faced with the requirement to cleanse mailboxes. The pen may be mightier than the sword, but a single mouse-click can destroy products of inestimable value.

The ease and simplicity of e-mail has altered the fundamental psychology of communication - and of confidentiality. Most of us are oblivious to the reality that e-communication violates the confidentiality intrinsic to conversations: e-mail records (even deleted ones) can be subpoenaed by courts of law. Other people's computers can easily be used to send or receive e-mail, or to access the Internet. The potential for violations of confidentiality and for impersonation is all too obvious, and examples of 'e-fraud' abound.

Even in honest use, the immediacy of e-mail breeds poor communication and can rapidly escalate conflict. The facile nature of the medium encourages a lack of

sensitivity, and the false assumption that such communication is private and confidential can lead to situations such as that of the recovery of e-mails questioning the safety of the US space shuttle's re-entry written days before the recent disaster.

Electronic communication is surely here to stay. Its simplicity and enormous speed will ultimately send other modes of communication close to extinction. But IT solutions to the many problems it brings are long overdue. Most serious is the potential for the loss of records that may have immense historical value. For a start, academic (and other) institutions should require hard copies of certain categories of electronically generated information to be kept — with appropriate authentication. Errol C. Friedberg, Herbert K. Hagler, Kevin J. Land Department of Pathology, University of Texas Southwestern Medical Center, Dallas, TX 75390, USA

\section{Britannica: source of a passion for physics}

Sir - In his obituary of US physicist Julian Schwinger (Nature 370, 600; 1994), David S. Saxon wrote: "He once remarked that he had been reading straight through the Encyclopaedia Britannica and when he came to the letter P and to physics, that was it."

In another memorial article, Paul C. Martin and Sheldon L. Glashow wrote "Armed with the 11th edition of the Encyclopaedia Britannica and the books and journals in nearby libraries, Schwinger set himself apart from the establishment of teachers, textbooks and assignments."

(Physics Today 48 (10), 40-46; 1995).

These descriptions raised my interest in the contents of the famous 11th edition of the Encyclopaedia Britannica (1910), which also influenced the physicist Freeman Dyson profoundly (see S. S. Schweber QED and the Men Who Made It: Dyson, Feynman, Schwinger, and Tomonaga 479; Princeton Univ. Press, 1994).

After examining this edition, I found to my surprise that 'physics' is not an entry word. This is particularly strange as this edition was published a decade and a half after the great discoveries that marked the beginning of modern physics. In contrast, both 'chemistry' and 'physiology' are entry words, with many depictions of these two sciences. Was Schwinger's account of his early commitment to physics perhaps either a joke or a fable?

Of course, many entry words regarding the science of physics were included and embedded in different volumes of the Encyclopaedia Britannica, so one could well believe that young Schwinger learnt physics from the entire set.

\section{Min-Liang Wong}

Department of Veterinary Medicine, College of Veterinary Medicine, National Chung-Hsing University, Taichung 402, Taiwan

\section{Britannica: which edition inspired Schwinger?}

Sir — Regarding Julian Schwinger's story of finding his subject by reading through the Encyclopaedia Britannica and stopping at 'Physics': an entry entitled 'Physics' appeared in every edition, beginning with the first in 1768 , until the 8th (1852-60). In the 9th (1875-89) it was replaced with 'Physical Sciences' (by James Clerk Maxwell), from a paper "Remarks on the classification of the physical sciences". 'Physics' as an entry title did not appear in the 11th or 12th, but it did appear in the 13th (1926), and in the modern sense, whereas previously it was an alternative name for 'natural philosophy' or 'the science of nature'. The author was Robert Andrews Millikan. The 12 th edition was actually the 11 th with some supplementary volumes, as was the 13th. Anita Wolff Senior Editor, Encyclopaedia Britannica, 310 South Michigan Avenue, Chicago, Illinois 60604, USA 